\title{
What should we do about vaccine hesitancy?
}

\author{
Fiona Godlee editor in chief
}

The BMJ

With the resurgence of measles-a disease we thought had been all but conquered-gaps in vaccination coverage are gaining increasing public attention. If vaccination rates continue their downward trajectory measles may once again become endemic. What should we do in the face of growing "vaccine hesitancy," now listed as one of the World Health Organization's 10 threats to global health (www.who.int/emergencies/ten-threats-toglobal-health-in-2019)? Should we blame the parents, censor social media, enforce vaccination through school exclusion, or penalise non-vaccination through fines or loss of benefits?

These are just some of the approaches found around the world and championed by Eleanor Draeger in this week's Head to Head debate (doi:10.1136/bmj.12359). To get vaccination coverage back to levels that will restore herd immunity, the UK should legislate to ban unvaccinated children from school, she says. Helen Bedford and David Elliman aren't convinced. It's not vaccine hesitancy that's driving the decline in the UK, they say, but the many small barriers that can make it hard for parents to get their children vaccinated. And even with better services and easier access, making vaccination mandatory could be counterproductive, they say, as it would damage the public's trust in health professionals and disproportionately affect children of poorer parents.

Maintaining civil discourse in the face of anti-vax rhetoric remains a crucial challenge. Last month David Oliver asked whether it's possible or even wise to engage (doi:10.1136/bmj.
12244). And on BMJ Opinion Peter Hotez described how poorly funded public health agencies can't even begin to compete with the quantity of populist pseudoscience being shared through social media (http://bit.ly/2JT51dj).

What more can be done to assuage legitimate safety concerns? Rebecca Chandler of WHO's Uppsala Monitoring Centre says we need better pharmacovigilance (doi:10.1136/bmj.12268). Taking as her example reports of a possible link between HPV vaccine and postural orthostatic tachycardia syndrome, she argues that current systems can't easily detect rare or poorly characterised adverse responses to vaccination. What's more, they are designed for regulators and policymakers rather than those seeking to advance scientific knowledge about how vaccines cause adverse events. She sees great promise in machine learning for better pattern recognition, as well as in our growing understanding of individual variation in immune responses to vaccination. But without better approaches to pharmacovigilance, we can't expect to earn the public's trust, she says.

Mass vaccination has achieved some of the greatest advances in human health. But it is also one of the greatest tests of societal cohesion and public trust. Confronted with the prospect of declining public trust and the re-emergence of serious preventable diseases, we have urgent choices to make. Rather than coercion and censorship, let's choose science, education, access, civil discourse, and debate. 